\title{
ROLES OF TYPE VI COLLAGEN AND DECORIN IN HUMAN MESENCHYMAL STEM CELL BIOPHYSICS DURING CHONDROGENIC DIFFERENTIATION
}

\author{
J.D. Twomey ${ }^{1}$, P.I. Thakore ${ }^{1}$, D.A. Hartman ${ }^{1}$, E.G.H. Myers ${ }^{1}$ and A.H. Hsieh ${ }^{1,2, *}$ \\ ${ }^{1}$ Fischell Department of Bioengineering, University of Maryland, College Park, MD 20740, USA \\ ${ }^{2}$ Department of Orthopaedics, University of Maryland, Baltimore, MD 21201, USA
}

\begin{abstract}
Human mesenchymal stem cells (hMSCs) induced towards chondrogenesis develop a pericellular matrix (PCM), rich in type VI collagen (ColVI) and proteoglycans such as decorin (DCN). Individual PCM protein functions still need to be elucidated to fully understand the mechanobiological role of this matrix. In this study we identified ColVI and DCN as important contributors in the mechanical function of the PCM and as biochemical modulators during chondrogenesis through targeted knockdown using shRNA lentiviral vectors. Gene expression, western blotting, immunofluorescence and cell deformation analysis were examined at 7, 14 and 28 days post chondrogenic induction. ColVI and DCN knockdown each affected gene expression of acan, bgn, and sox 9 during chondrogenesis. ColVI was found to be of central importance in resisting applied strains, while DCN knockdown had strain dependent effects on deformation. We demonstrate that by using genetic engineering to control the biophysical microenvironment created by differentiating cells, it may be possible to guide cellular mechanotransduction.
\end{abstract}

Keywords: Pericellular matrix, human mesenchymal stem cell, type VI collagen, decorin, chondrogenesis, RNA interference.

*Address for correspondence:

Adam H. Hsieh

Jeong H. Kim Engineering Building

Room 3242

College Park, MD 20742, USA

Telephone Number: 301.405 .7397

FAX Number: 301.314 .6868

Email: hsieh@umd.edu

\section{Introduction}

The use of human mesenchymal stem cells (hMSCs) in regenerative medicine has the potential to repair damage in load bearing tissues such as articular cartilage (Alexopoulos et al., 2003; Guilak et al., 2006), reducing the need for autologous chondrocytes. During chondrogenesis, hMSCs develop a pericellular matrix (PCM) that is rich in type VI collagen (ColVI) (Poole et al., 1992; Wiberg et al., 2003; Zhang et al., 2011), fibronectin (Wiberg et al., 2003; DiMicco et al., 2007; Connelly et al., 2008), hyaluronan (Knight et al., 1998; Zhang et al., 2011), and proteoglycans (PGs) such as aggrecan, decorin (DCN), and biglycan (BGN) (Wiberg et al., 2003; Connelly et al., 2008). This thin 2-6 $\mu \mathrm{m}$ layer of matrix acts as both a biomechanical buffer (Knight et al., 1998; Lee et al., 2010), controlling the amount of deformation applied to the cell (Knight et al., 2001; Choi et al., 2007; Vigfusdottir et al., 2010), as well as a biochemical conductor, presenting the cell with growth factors and signalling molecules (Chen et al., 2009; Vigfusdottir et al., 2010; Zhang et al., 2011). The PCM is believed to modulate the mechanoresponsiveness of chondrocytes and chondrogenic hMSCs, but the exact roles of the individual PCM proteins has yet to be determined.

In articular cartilage, type VI collagen is found exclusively in the PCM surrounding chondrocytes (Poole et al., 1992; DiMicco et al., 2007) and is maintained through low levels of transcription, forming a microfibrillar network once secreted by the cell (Engvall et al., 1986; Poole et al., 1992; Wiberg et al., 2003; DiMicco et al., 2007; Vigfusdottir et al., 2010). ColVI's interactions with the cell membrane at varying locations make it a primary candidate as a mechanotransducer (Poole et al., 1992; Doane et al., 1998; Loeser et al., 2000). This complex network assembles and integrates with the surrounding extracellular matrix (ECM) through interactions with type II collagen, aggrecan, and hyaluronan with aid from PGs such as DCN, BGN, as well as type IX collagen (Engvall et al., 1986; Keene et al., 1988; Bidanset et al., 1992; Poole et al., 1992; Wiberg et al., 2003; Nareyeck et al., 2004; Connelly et al., 2008).

The PCM has shown direct control over cellular phenotype, as chondrocytes isolated without a PCM exhibit a phenotypic change characterised by greater collal and lower col2al expression than those isolated with a native PCM (Vonk et al., 2010). The metabolic activity of hMSCs undergoing chondrogenic differentiation changes with PCM development and is also different from that of mature chondrocytes (DiMicco et al., 2007; Connelly et al., 2008). Therefore, it is believed that the PCM directly 
surrounding chondrogenic hMSCs is temporally changing and complex, with the microenvironment composition influencing chondrogenic potential and response (Knight et al., 2001; DiMicco et al., 2007; Vonk et al., 2010).

Biomechanically, the developing PCM surrounding both mature chondrocytes and differentiating hMSCs has been found to reduce cell deformation in hydrogels under applied compression (Knight et al., 1998; Lee et al., 2010; Vigfusdottir et al., 2010). Protein deposition and biosynthesis progressively alter PCM material properties, causing increases in stiffness and elastic-like response (Lee et al., 2010). The ability of the PCM to help articular chondrocytes resist deformation has been shown to depend on hyaluronan during initial PCM assembly, and speculated to depend on collagen and other matrix components at later time points (Knight et al., 1998). Native chondrocytes with their PCM, which have been termed chondrons, exhibit a unique viscoelastic response to compression due to mismatched stiffnesses among the chondrocyte, its PCM, and the cartilage ECM (Guilak et al., 1999).

Toward an improved understanding of how individual components of the PCM biochemically and biophysically regulate hMSCs during chondrogenesis, our current study sought to elucidate the roles of ColVI and DCN in resisting cellular deformation as well as their relationships with other PCM gene expression. We determined the function of these specific proteins through targeted subtraction using shRNAmediated RNA interference (RNAi) (Stewart et al., 2003). Overall, we found that knockdown of ColVI and DCN differentially altered PCM accumulation, expression of other PCM components, and cell deformation in hydrogels. These results demonstrate that the microenvironment of chondrogenically differentiating hMSCs can be controlled using genetic engineering techniques to modulate PCM composition.

\section{Materials and Methods}

\section{Cell culture}

Passage 2 hMSCs were obtained from Lonza (Walkersville, MD, USA) and were expanded in monolayer according to the manufacturer's protocols, sub-culturing with growth media (GM): high glucose DMEM (HG-DMEM) (Gibco/ Invitrogen, Carlsbad, CA, USA) supplemented with $10 \%$ foetal bovine serum (FBS) (Gibco), $100 \mathrm{U} / \mathrm{mL}$ penicillin/ streptomycin (Gibco), 1 \% MEM non-essential amino acids (Gibco), and $4 \mathrm{mM}$ L-Glutamine (Invitrogen). Cells were cultured in monolayer at $37^{\circ} \mathrm{C}$ with $5 \% \mathrm{CO}_{2}$, with media changes three times per week until passage 4 or 5 , at which point they were used experimentally. Experimental and control groups were lifted from monolayer with $0.25 \%$ trypsin-EDTA (Gibco), centrifuged at $600 \mathrm{x}$ g for $5 \mathrm{~min}$ before being re-suspended in a $2 \%(\mathrm{w} / \mathrm{v})$ alginate solution (Research Products International, Mount Prospect, IL, USA) at a concentration of $2.0 \times 10^{6}$ cells $/ \mathrm{mL}$. The cellalginate solution was expelled drop-wise into a $102 \mathrm{mM}$ $\mathrm{CaCl}_{2}$ bath using a 22 gauge needle and allowed to cure for $10 \mathrm{~min}$. Alginate-hMSC beads were cultured in either GM or chondrogenic induction media (CM) containing HG-
DMEM, ITS Universal Culture Supplement Premix (BD Biosciences, San Jose, CA, USA), $10 \mathrm{nM}$ dexamethasone, $50 \mu \mathrm{g} / \mathrm{mL}$ L-ascorbic acid (Sigma-Aldrich, St. Louis, MO, USA), $40 \mu \mathrm{g} / \mathrm{mL}$ L-Proline (Sigma), $100 \mathrm{U} / \mathrm{mL}$ penicillin/streptomycin, $1 \mathrm{mM}$ sodium pyruvate, $0.584 \mathrm{~g} /$ $\mathrm{mL}$ L-glutamine, and $10 \mathrm{ng} / \mathrm{mL}$ TGF- $\beta 3$ (Lonza). Beads were cultured for 1,2 , or 4 weeks post seeding at $37^{\circ} \mathrm{C}$ with $5 \% \mathrm{CO}_{2}$.

\section{Lentivirus preparation}

21-23 nt shRNA sequences were designed using RNAi Codex (web ref. 1) from either human col6al [Gen Bank: NM_001848] or human dcn [GenBank: NM_001920] mRNA. Stem-loop-stem shRNA constructs were created, annealed to create complimentary oligos, and cloned into a lentiviral vector containing a blasticidin resistance gene with a U6 promoter and a Pol III termination sequence. The BLOCK-iT U6 RNAi Entry Vector Kit and the BLOCK-iT Lentiviral RNAi Expression System (Invitrogen, Carlsbad, CA) were used to generate shRNA constructs and packaged into a replication-deficient lentivirus (denoted shColVIA-E and shDen A-F) using 293FT cells, Lipofectamine 2000 and a manufacturer-supplied packaging mix (Invitrogen). The chosen sequences are listed in Table 1. A lentiviral expression vector containing the gene for GFP was prepared in parallel using the Vivid Colors pLenti6.2GW/EmGFP kit (Invitrogen) for a lentiviral control. Virus-containing supernatants were harvested $72 \mathrm{~h}$ post transfection and stored at $-80^{\circ} \mathrm{C}$ until used experimentally.

\section{shRNA transduction}

For initial screening trials to identify optimal shRNA viral vectors, hMSCs were seeded at 60,000 cells/ well in tissue culture treated polystyrene 6-well plates. Monolayers of hMSCs were infected with lentiviral particles in the presence of $6 \mu \mathrm{g} / \mathrm{mL}$ Polybrene (Sigma) to aid in transduction efficiency. Each well was incubated with $100 \mu \mathrm{L}$ of either an shColVI (A-E) or an shDen (A-F) vector for $24 \mathrm{~h}$. Cell monolayers were then washed and maintained in GM culture without selection for $1,5,8$ or 14 days post infection, at which point gene expression was assessed.

In subsequent experiments, each well was incubated with either shColVI-D, shDcn-F (identified from our screen; hereafter labelled shColVI and shDcn, respectively), or the GFP control vector at a multiplicity of infection (MOI) of 1 for $24 \mathrm{~h}$. Titered viral concentrations for an MOI of 1 were determined through a Quant-IT PicoGreen Assay (Invitrogen). After virus removal, cells were incubated with GM containing $12 \mu \mathrm{g} / \mathrm{mL}$ of blasticidin for $24 \mathrm{~h}$, to select a pure population of transduced cells. We performed extensive preliminary sensitivity tests to identify this shorthigh intensity treatment for minimising adverse effects of blasticidin culture. Blasticidin-free shRNA-infected control and non-infected control hMSCs were cultured in parallel. Some hMSCs were cultured for $1,4,7$ or 14 days post-infection for gene expression studies. Some blasticidin-selected pure populations were trypsinised for alginate bead culture $24 \mathrm{~h}$ post selection (day 0 ). 
Table 1: Sense and anti-sense hairpin sequences for shRNA constructs against colbal or dcn.

\begin{tabular}{|c|c|c|}
\hline & & 5'-"stem-loop-stem"-3' sense and 3'-"stem-loop-stem"-5' anti-sense sequences \\
\hline \multirow{10}{*}{$\sum_{\frac{1}{0}}$} & \multirow{2}{*}{ A } & 5'-CACCGCCTTTGGACTGAAAGGAGACGAATCTCCTTTCAGTCCAAAGG-3' \\
\hline & & 3'-CGGAAACCTGACTTTCCTCTGCTTAGAGGAAAGTCAGGTTTCCAAAA-5' \\
\hline & \multirow{2}{*}{ B } & 5'-CACCGTGGGCATCAAAGACGTGTTTCGAAAAACACGTCTTTGATGCCCAC-3' \\
\hline & & 3'-CACCCGTAGTTTCTGCACAAAGCTTTTTGTGCAGAAACTACGGGTGAAAA-5' \\
\hline & \multirow{2}{*}{$\mathrm{C}$} & 5'-CACCGCAAAGTCAAGTCCTTCACCAACGAATTGGTGAAGGACTTGACTTTG-3' \\
\hline & & 3'-CGTTTCAGTTCAGGAAGTGGTTGCTTAACCACTTCCTGAACTGAAACAAAA-5' \\
\hline & \multirow{2}{*}{$\mathrm{D}$} & 5'-CACCGCGGAGACGATAACAACGACATCGAAATGTCGTTGTTATCGTCTCCG-3' \\
\hline & & 3'-CGCCTCTGCTATTGTTGCTGTAGCTTTACAGCAACAATAGCAGAGGCAAAA -5' \\
\hline & \multirow{2}{*}{$\mathrm{E}$} & 5'-CACCGCTGTGTCTTACTAGAAACAACGAATTGTTTCTAGTAAGACACAGC-3' \\
\hline & & 3'-CGACACAGAATGATCTTTGTTGCTTAACAAAGATCATTCTGTGTCGAAAA-5' \\
\hline \multirow{12}{*}{ 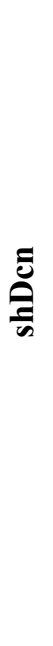 } & \multirow{2}{*}{ A } & 5'-CACCGCTACTAGAGATATTCTTATCGAAATAAGAATATCTCTAGTAG-3' \\
\hline & & 3'-CGATGATCTCTATAAGAATAGCTTTATTCTTATAGAGATCATCAAAA-5' \\
\hline & \multirow{2}{*}{ B } & 5'-CACCGCAAATTTCCAGTTTAAGTACGAATACTTAAACTGGAAATTTG-3' \\
\hline & & 3'- CGTTTAAAGGTCAAATTCATGCTTATGAATTTGACCTTTAAACAAAA-5' \\
\hline & \multirow{2}{*}{$\mathrm{C}$} & 5'-CACCGCCAGGTTGTCTACCTTCATAACGAATTATGAAGGTAGACAACCTGG-3' \\
\hline & & 3'- CGGTCCAACAGATGGAAGTATTGCTTAATACTTCCATCTGTTGGACCAAAA-5' \\
\hline & \multirow{2}{*}{$\mathrm{D}$} & 5'-CACCGCGACTTTATCTGTCCAAGAATCGAAATTCTTGGACAGATAAAGTCG-3' \\
\hline & & 3'-CGCTGAAATAGACAGGTTCTTAGCTTTAAGAACCTGTCTATTTAAGCAAAA-5' \\
\hline & \multirow{2}{*}{$\mathrm{E}$} & 5'-CACCGCCATTCAACTCGGAAACTATCGAAATAGTTTCCGAGTTGAATGGC-3' \\
\hline & & 3'-CGGTAAGTTGAGCCTTTGATAGCTTTATCAAAGGCTCAACTTACCGAAAA-5' \\
\hline & \multirow{2}{*}{$\mathrm{F}$} & 5'-CACCGCCGTTTCAACAGAGAGGCTTACGAATAAGCCTCTCTGTTGAAACGG-3' \\
\hline & & 3'-CGGCAAAGTTGTCTCTCCGAATGCTTATTCGGAGAGACAACTTTGCCAAAA-5' \\
\hline
\end{tabular}

Table 2: Sequences of primers used for qRT-PCR.

\begin{tabular}{|c|c|c|}
\hline Gene & Forward and Reverse sequences & GenBank accession no. \\
\hline \multirow{2}{*}{ col6a1 } & 5'-CTACACCGACTGCGCTATCA-3' & \multirow{2}{*}{ NM_001848 } \\
\hline & 5'-GCCACCGAGAAGACTTTGAC-3' & \\
\hline \multirow{2}{*}{ col6a 2} & 5'-ACCGAGATCAACCAGGACAC-3' & \multirow{2}{*}{ NM_001849 } \\
\hline & 5'-GGTCTCCCTGTCTTCCCTTC 3' & \\
\hline \multirow{2}{*}{ col6a3 } & 5'-GCCAACCATTGTCACACAAG-3' & \multirow{2}{*}{ NM_004369 } \\
\hline & 5'-TTCAGGCCTCACAGTGTCTG-3' & \\
\hline \multirow{2}{*}{$d e n$} & 5'-AATTGAAAATGGGGCTTTCC-3' & \multirow{2}{*}{ NM_001920 } \\
\hline & 5'-GCCATTGTCAACAGCAGAGA-3' & \\
\hline \multirow{2}{*}{ gapdh } & 5'-GAGTCAACGGATTTGGTCGT-3' & \multirow{2}{*}{ NM_002046 } \\
\hline & 5'-TTGATTTTGGAGGGATCTCG-3' & \\
\hline \multirow{2}{*}{ acan } & 5'-ACAGCTGGGGACATTAGTGG-3' & \multirow{2}{*}{ NM_001135 } \\
\hline & 5'-GTGGAATGCAGAGGTGGTTT-3' & \\
\hline \multirow{2}{*}{$\operatorname{sox} 9$} & 5'-AGTACCCGCACTTGCACAAC-3' & \multirow{2}{*}{ NM_000346 } \\
\hline & 5'-CGTTCTTCACCGACTTCCTC-3' & \\
\hline \multirow{2}{*}{$b g n$} & 5'-ACCTCCCTGAGACCCTGAAT-3' & \multirow{2}{*}{ NM_001711 } \\
\hline & 5'-CTGGAGGAGCTTGAGGTCTG-3' & \\
\hline \multirow{2}{*}{$\operatorname{col9a2}$} & 5'-GCGGATTTCCTGTGTCCAA-3' & \multirow{2}{*}{ NM_001852 } \\
\hline & 5'-CCGCATGCCCCTTCACT-3' & \\
\hline
\end{tabular}


A
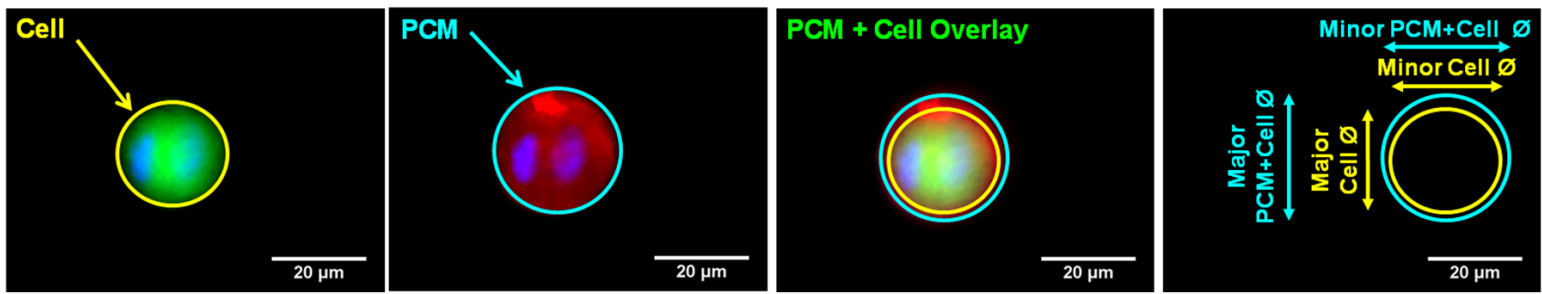

B
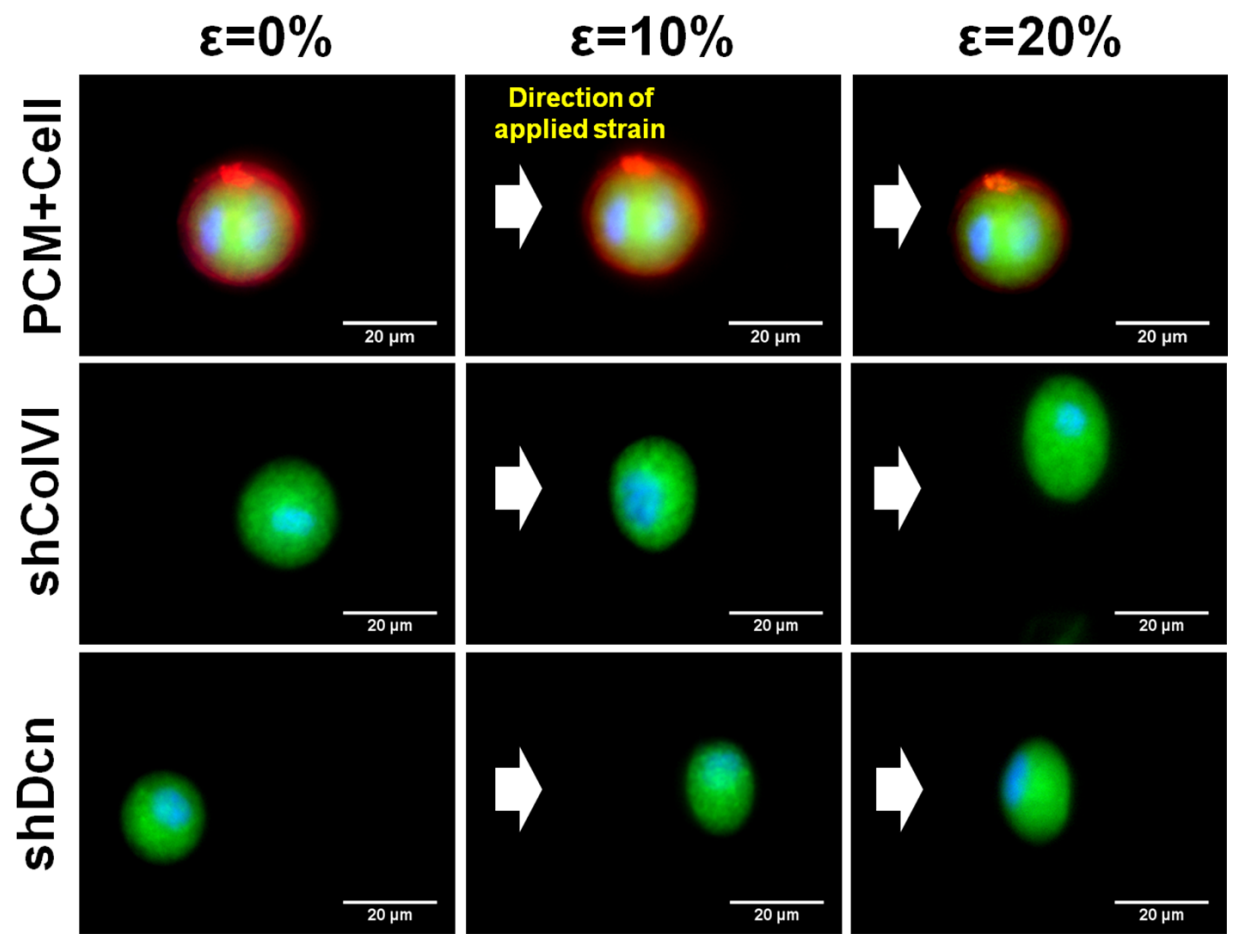

Fig. 1. Representative images of aspect ratio analysis under static applied strain. Cells are stained with CMFDA (green), the PCM with 6ROX (red), and nuclei with DAPI (blue). (A) A yellow ellipse is shown around the cell and a blue ellipse is shown around the PCM to indicate how the major and minor diameters of the cell and the $\mathrm{PCM}+\mathrm{Cell}$ were obtained. (B) PCM+Cell of CM-hMSCs, shColVI- and shDen- transduced cells at day 14 under static applied strain of 0,10 and $20 \%$. The white arrows indicate the direction of applied compression. All scale bars represent $20 \mu \mathrm{m}$.

\section{Cell viability}

To determine the viability of cells in alginate beads, at each time point per sample two or three beads were centrally cut and transferred to a working solution of 5-chloromethylfluorescein diacetate (CMFDA) and ethidium homodimer-1 (EthD-1) (Invitrogen) and counterstained with 4',6-diamidino-2-phenylindole (DAPI) (Invitrogen). From the cut face of the beads, ten confocal fluorescence image stacks of $30 \mu \mathrm{m}$ depth were taken at 100x magnification with a Nipkow (spinning) disk-equipped Olympus IX81 microscope. Viability was assessed on sections using channel separation and threshold particle analysis using ImageJ (NIH). All results are expressed as a percentage of total cells that are viable (green only).

\section{Gene expression}

Gene expression was quantitatively assessed as previously described (Rastogi et al., 2009). At each time point, cells (shColVI, shDcn, and GFP transduced, along with a non- infected hMSC control) were subsequently released with a $100 \mathrm{mM}$ sodium citrate, $30 \mathrm{mM}$ EDTA solution, spun down and re-suspended in a buffer solution containing $\beta$-Mercaptoethanol (Fisher Scientific, Pittsburgh, PA, USA) before flash freezing. Total RNA was isolated (RNeasy Micro, Qiagen, Valencia, CA, USA) and reverse transcribed. Gene expression was analysed using qRTPCR (MyiQ System, BioRad, Hercules, CA, USA) with primers designed for human genes (Table 2) using Primer3 software (web ref. 2). All primer amplification efficiencies were determined using linear regression efficiency methods and were determined to be between $91.2 \%$ and $108.2 \%$ $\left(\mathrm{R}^{2}>0.99\right)$ (Rutledge and Cote, 2003). Expression levels for acan, sox9, bgn, col6a1, col6a2, col6a3, dcn, col9a2 and housekeeping gene $G A P D H$ were analysed using the $\triangle \triangle \mathrm{Ct}$ method. Threshold cycle $(\mathrm{Ct})$ values were averaged and $\Delta \mathrm{Ct}$ was calculated by subtracting the average $\mathrm{Ct}$ values of $G A P D H$ from those of the gene of interest (e.g. $\Delta \mathrm{Ct}_{\text {colbal }}=\mathrm{Ct}_{\text {colbal }}-\mathrm{Ct}_{\text {GAPDH }}$ ). $\Delta \Delta \mathrm{Ct}$ for each gene of interest was determined by subtracting the designated 
control $\Delta \mathrm{Ct}$ from the experimental $\Delta \mathrm{Ct}$ at each time point (e.g. $\left.\Delta \Delta \mathrm{Ct}_{\text {col6al, } \mathrm{D} 7}=\left(\Delta \mathrm{Ct}_{\text {coloal, shColvI, D7 }}-\Delta \mathrm{Ct}_{\text {col6al, } \mathrm{GFP}, \mathrm{D} 7}\right)\right)$. Relative gene expression levels (fold difference) were computed though the exponential relation $2^{-\Delta \Delta \mathrm{Ct}}$ (Livak and Schmittgen, 2001). Data are shown as average values of the range of calculated fold differences $\left(2^{-\Delta \Delta C t+S D}\right.$ and $\left.2^{-\Delta \Delta \mathrm{Ct}-\mathrm{SD}}\right) \pm$ range.

\section{Western blotting}

To quantify protein translation, cells were released from alginate as described above. Samples were re-suspended in a lysis buffer (50 mM HEPES (Fisher), $150 \mathrm{mM}$ sodium chloride (Fisher), $1 \%$ Triton X-100 (Ricca Chemical Compant, Arlington, TX, USA), 1 mM EDTA (Fisher), $10 \mathrm{mM}$ Na-pyrophosphate (Fisher), $10 \%$ glycerine (Fisher)) with a 1:100 concentration of protease inhibitor cocktail (Fisher Scientific) to precipitate out the proteins. A portion of the protein supernatant was separated for a modified Lowry assay to determine protein concentration using a Folin-Phenol colour reaction detected by a ND1000 Spectrophotometer (Nanodrop, Wilmington, DE). The remaining supernatant was mixed 1:1 with a loading buffer (13\% (v/v) Tris-HCl, $20 \%$ (v/v) glycerol, $4.6 \%$ (w/v) SDS, $0.02 \%(\mathrm{w} / \mathrm{v})$ bromophenol blue, $200 \mathrm{mM}$ dithiothreitol). SDS-PAGE was performed using pre-cast Criterion Tris- $\mathrm{HCl}$ gels (BioRad) using equal amounts of protein from each sample. Proteins were detected with rabbit IgG antibodies targeting human $\beta$-actin (R-22; Santa Cruz Biotechnology, Dallas, TX, USA), $\alpha 1(\mathrm{VI})$ (H-200; Santa Cruz), and decorin (H80; Santa Cruz). For decorin detection, blots were incubated with $1 \mathrm{U} / \mathrm{mL}$ chondroitinase ABC from Proteus vulgaris to digest dermatan-sulphate GAG chains from the decorin core protein (C2905, SigmaAldrich) for $3 \mathrm{~h}$ and washed with DPBS before blocking. Positive controls of CCD-1064Sk Cell Lysate (Santa Cruz) for $\beta$-actin and $\alpha 1$ (VI), and 293 Lysate (Santa Cruz) for DCN were run simultaneously to ensure valid detection. Semi-quantitative analysis was performed using ImageJ to determine band intensities. Protein expression levels are shown relative to non-infected cells \pm standard error of the mean (SEM).

\section{Immunofluorescence}

For immunofluorescence visualisation, at each time point, alginate beads were sequentially fixed overnight in a $4 \%$ paraformaldehyde (PFA), $0.05 \mathrm{M}$ sodium cacodylate solution, equilibrated in $30 \%$ sucrose, embedded in Tissue-Tek O.C.T. compound (Sakura, Torrance, CA, USA), and frozen and maintained at $-80{ }^{\circ} \mathrm{C}$ until use. Frozen sections $(24 \mu \mathrm{m})$ were created using an HM550 series cryostat (Richard Allen Scientific, Kalamazoo, MI, USA) and placed on gelatin-coated slides (Electron Microscopy Services, Hatfield, PA, USA). For type VI collagen detection, sections were labelled with a rabbit IgG anti-human type VI collagen primary antibody (H-200; Santa Cruz). For decorin imaging, sections were incubated with $1 \mathrm{U} / \mathrm{mL}$ chondroitinase $\mathrm{ABC}$ for $2 \mathrm{~h}$ and washed with DPBS before blocking. Sections were then labelled with a rabbit IgG anti-human decorin primary antibody (H80; Santa Cruz). Sections were visualised with biotinylated anti-rabbit IgG secondary antibodies and Texas red- labelled streptavadin (Labvision/Thermo Fisher Scientific, Kalamazoo, MI, USA) and counterstained with DAPI. Fluorescence images were taken at 400x magnification with a Nipkow (spinning) disk-equipped Olympus IX81 microscope.

\section{Cellular deformation}

For aspect ratio measurements under static compression, cells were isolated and re-embedded with their PCMs intact in new hydrogel constructs, to eliminate effects of changing alginate bead properties, as previously described (Vigfusdottir et al., 2010). After cells were released from alginate using sodium citrate and EDTA, they were incubated with CMFDA to stain cell cytoplasm, 6-Carboxy-X-Rhodamine (6-ROX; Invitrogen) to stain non-specifically for the PCM and DAPI. Stained cells were then resuspended in $2 \%(\mathrm{w} / \mathrm{v})$ alginate and pipetted into a $6 \mathrm{~mm} \times 6 \mathrm{~mm} \times 10 \mathrm{~mm}$ mould with a $10 \mu \mathrm{m}$ porous membrane and Whatman thick blot paper (Biorad) paper attached to the top and bottom faces. The moulds were immersed in $102 \mathrm{mM} \mathrm{CaCl}_{2}$ for $2 \mathrm{~h}$ to cure (Wang et al., 2011). After curing, constructs were placed into a custom made micrometer-controlled deformation device (Vigfusdottir et al., 2010) and imaged at $0 \%, 10 \%$ and $20 \%$ uni-axial bulk strain. Fluorescence images were acquired at 400x magnification. Major and minor cell diameters as well as the stained PCM (PCM+Cell) diameters were measured using Image $\mathrm{J}(\mathrm{NIH})$ and used to calculate aspect ratios ( $\mathrm{AR}=$ minor cell diameter/ major cell diameter), as depicted in Fig. 1A. Representative images of PCM+Cell (non-infected), shColVI-transduced, and shDcn-transduced cells at 0,10 and $20 \%$ applied strain are shown in Fig. 1B. Normalised ARs (NAR) were calculated at each strain for each sample's population of average deformed AR compared to its un-deformed AR. Data are shown as NAR \pm SEM.

\section{Statistical analysis}

Statistical analyses for cell viability, gene expression, western blotting and aspect ratios were performed using one-way ANOVA. Tukey's HSD post hoc tests were performed for pairwise comparisons. All computations were performed using JMP7 (Cary, NC) with statistical significance set to $\alpha<0.05$ or $\alpha<0.01$ as indicated in results.

\section{Results}

\section{Lentiviral vectors can induce efficient shRNA- mediated RNAi in hMSCs}

Initial screening was conducted on hMSC monolayers to identify optimal shRNA constructs to use for subsequent experiments. Because each shRNA sequence was designed to target different locations along the mRNA strand, variation in knockdown due to mRNA folding was expected. We found that the shColVI D construct $(\mathrm{n}=2)$ was most effective in knocking down col6al transcript levels, resulting in decreases of up to $45 \%$ relative to non-infected hMSCs in monolayer over $14 \mathrm{~d}$. The shDcn $\mathrm{F}$ construct induced the greatest knockdown of $d c n$ 
Fig. 2. Knockdown of target genes and protein in samples cultured in CM. (A) Colbal and dcn relative gene expression as assessed by qRTPCR $(n \geq 3)(* p<0.05, * * p<0.01$ to non-infected and GFP-transduced hMSCs). Data are shown as average values of the range of calculated fold differences $\left(2^{-\Delta \Delta \mathrm{Ct}+\mathrm{SD}}\right.$ and $\left.2^{-\Delta \Delta \mathrm{Ct}-\mathrm{SD}}\right) \pm$ range. (B) ColVI and DCN protein expression as analysed by western blotting (D7, $n=2$; D14, $n=2$; D28, $n=4)$ in GFP and shColVI groups relative to non-infected CM-hMSCs at corresponding time points. Relative protein expression was calculated as the ratio of the shRNA-transduced cells (target protein/ $\beta$-actin) to non-infected CM-hMSCs (target protein/ $\beta$-actin). Data are represented as mean \pm SEM. (C) Representative western blotting at day 14 for $\alpha 1(\mathrm{VI}), \mathrm{DCN}$, and $\beta$-actin in non-infected CM-hMSCs, and GFP-, shColVI-, and shDentransduced cells. Positive controls $(+)$ were run in parallel.
A

shColVI knockdown

\section{shDen knockdown}
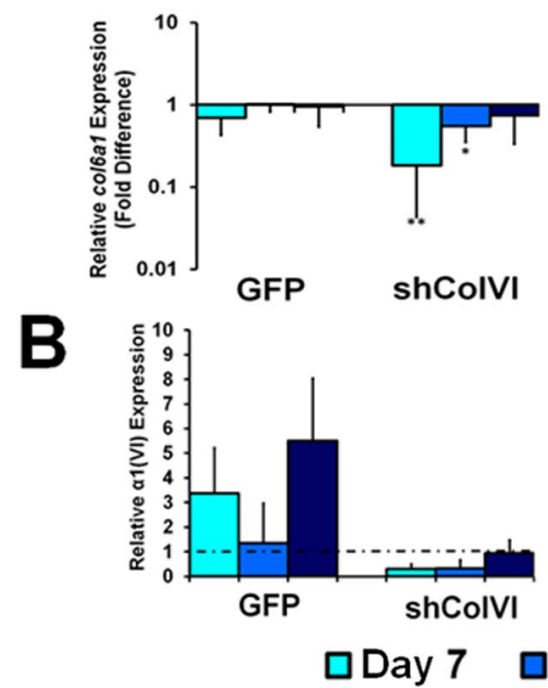

C
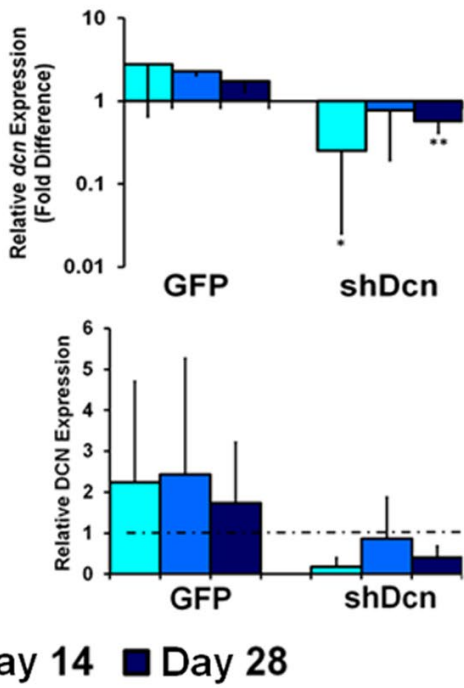

Day 14

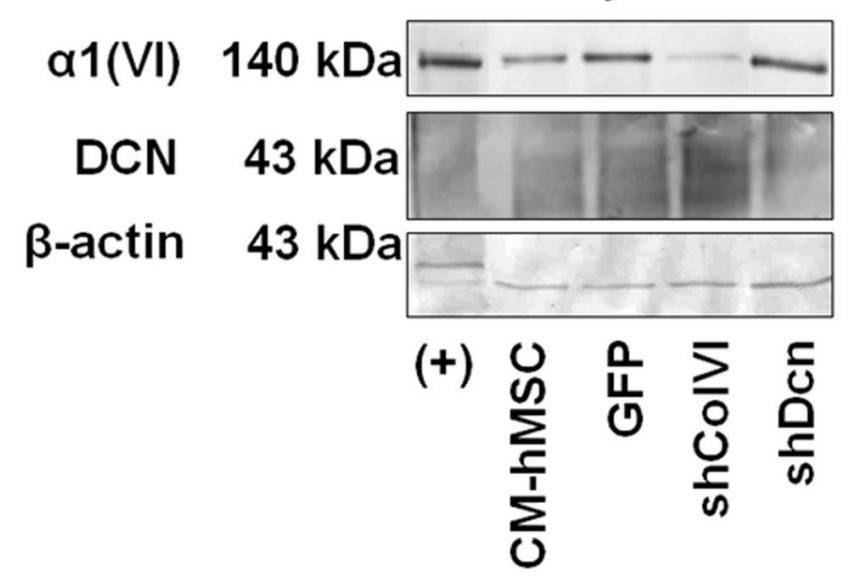

A

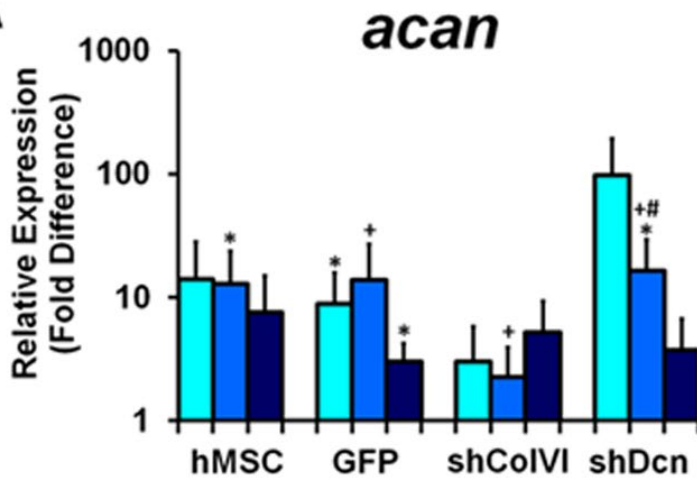
口Day
B

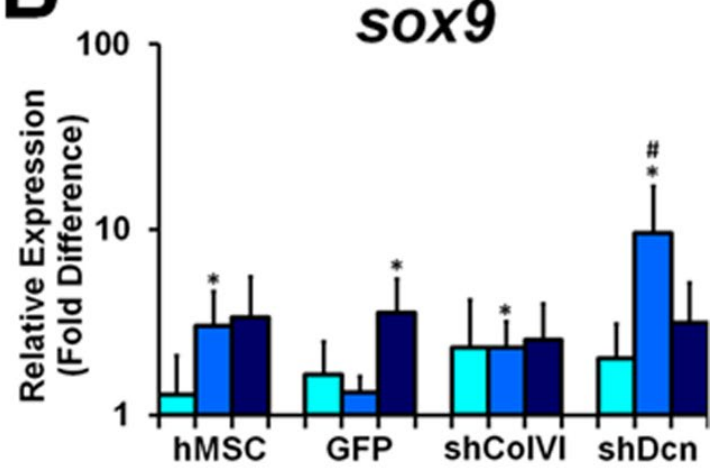

Day 14 Day 28

Fig. 3. Chondrogenic gene expression in control and knockdown cells. (A) Gene expression for acan and (B) sox9 in non-infected, and GFP-, shColVI- and shDen- transduced hMSCs in alginate bead culture $(n=3)$.All data represent $\mathrm{CM}$ relative to their same infection condition cultured in GM at 7, 14 and $28 \mathrm{~d}\left(\mathrm{e} . \mathrm{g} \Delta \Delta \mathrm{Ct}{ }_{\operatorname{acan} D 7}=\right.$ $\left.\Delta \mathrm{Ct}_{a c a n, C M, D^{7}}-\Delta \mathrm{Ct}_{a c a n, G M, D 7}\right)\left({ }^{*} p<0.05 \mathrm{CM}\right.$ culture relative to same condition same time point in $\mathrm{GM} ;+p<0.05$, relative to CM-hMSCs at same time point; $\# p<0.05$, relative to CM-GFP hMSCs at same time point; $n \geq 3$ ). Data are shown as average values of the range of calculated fold differences $\left(2^{-\Delta \Delta \mathrm{Ct}+\mathrm{SD}}\right.$ and $\left.2^{-\Delta \Delta \mathrm{Ct}-\mathrm{SD}}\right) \pm$ range. 

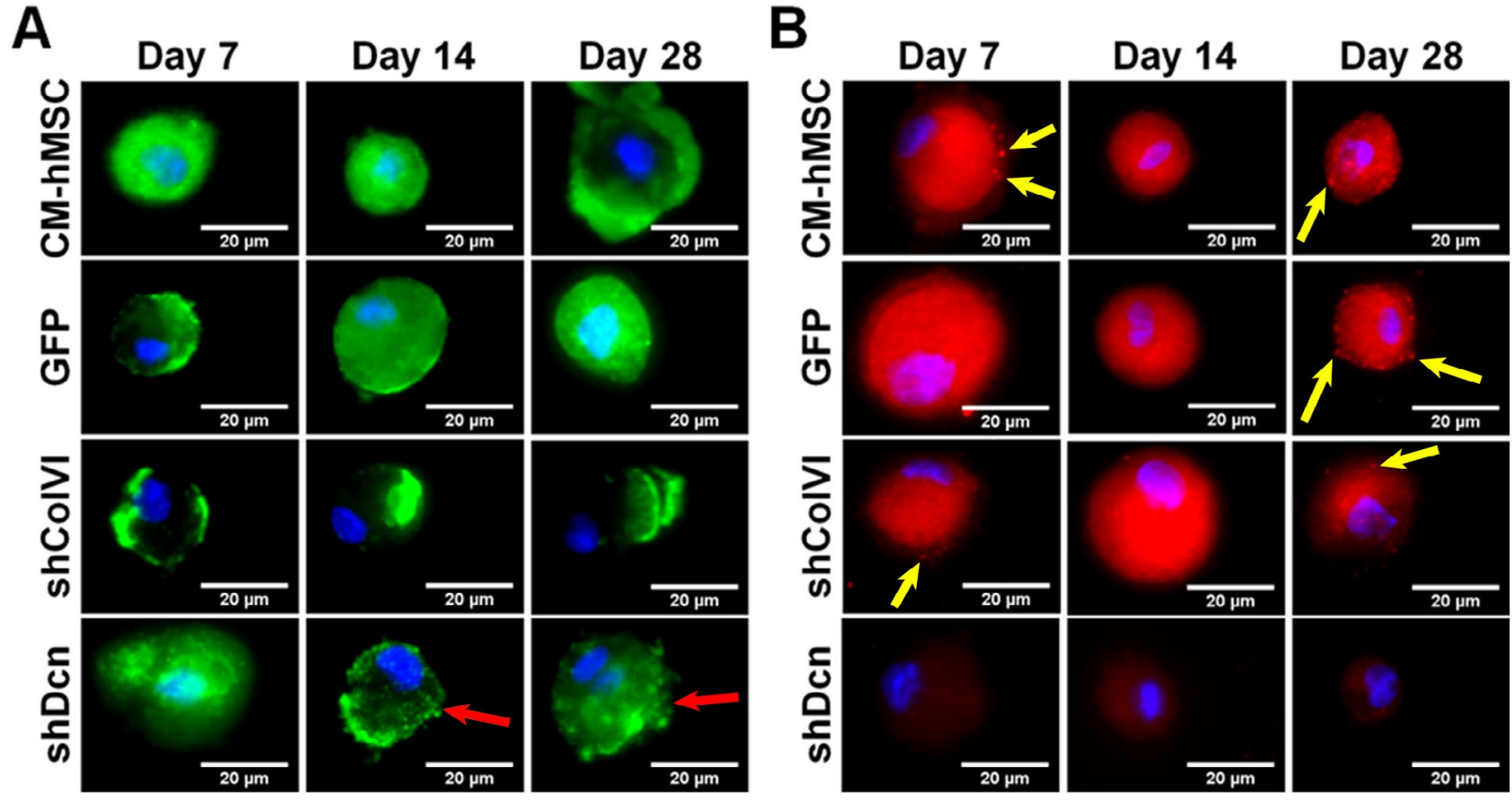

Fig. 4. Confocal microscopy visualisation of ColVI and DCN. (A) ColVI (green) immunofluorescence visualisation within the PCM of non-infected, and GFP-, shColVI-, and shDcn-transduced hMSCs cultured in alginate beads in $\mathrm{CM}$ at 7, 14 and $28 \mathrm{~d}$. Cell nuclei are stained with DAPI (blue). Red arrows point to punctate ColVI staining. All images were standardised to similar pixel intensity ranges for valid comparisons. Negative controls without primary or secondary labelling showed no ColVI staining (data not shown). (B) Equivalent data for DCN (red) immunofluorescence visualisation. Yellow arrows point to concentrated clusters of DCN around the cell membrane. Negative controls without primary or secondary labelling showed no DCN staining (data not shown). All scale bars represent $20 \mu \mathrm{m}$.

expression, with up to $91 \%$ silencing over $14 \mathrm{~d}(n=2)$. Other constructs were less effective in inducing RNAi and were, therefore, excluded from subsequent experiments.

Based on the screen, we performed experiments using blasticidin selection, to eliminate any non-transduced cells, and assessed gene silencing in hMSCs with shColVI D and shDen F constructs (hereafter labelled shColVI and shDen, respectively). The pure population of shColVI-transduced cells exhibited col6al expression that was significantly decreased by $67 \%$ at day $4(p<0.01, n \geq 2)$ and remained significantly depressed through $14 \mathrm{~d}(p<0.01, n \geq 2)$. Construct shDen achieved a significant $89 \%$ knockdown of $d c n$ expression at day 1 and maintained this diminished level over the entire $14 \mathrm{~d}$ ( $p<0.01$ for all time points, $n \geq 2$ ).

\section{Gene silencing of PCM proteins does not affect chondrogenic differentiation}

As in our previous studies (Vigfusdottir et al., 2010), we used alginate bead culture of hMSCs in TGF- $\beta 3$ supplemented chondrogenic induction media as a model system to study PCM formation during differentiation. To validate this approach for transduced cells, we verified knockdown of the target genes, quantified cell viability, and confirmed chondrogenic gene expression.

Significant target gene knockdown was achieved for both shRNA constructs, as assessed by qRT-PCR, relative to controls of non-infected hMSCs and of a lentivirus transduced GFP expression cassette (Fig.
2A). For shColVI-transduced cells, col61al knockdown remained statistically significant through $14 \mathrm{~d}$ but gradually diminished toward control levels by day 28 (Fig. 2A. day $7: 0.183 \pm 0.140, p<0.01$; day $14: 0.549 \pm 0.200, p<0.05$; day 28: $0.740 \pm 0.406)$. For shDcn-transduced cells, $d c n$ knockdown was statistically significant at 7 and $28 \mathrm{~d}$ (Fig. 2A. day 7: $0.253 \pm 0.228, p<0.05$; day 14: $0.769 \pm 0.575$; day 28: $0.574 \pm 0.161, p<0.01$ ) but not at $14 \mathrm{~d}$. Western blots demonstrated that protein levels mirrored the temporal variations we observed for transcript levels (Fig. $2 \mathrm{~B}$ and $2 \mathrm{C}$ ). Cells transduced with their respective silencing constructs produced less $\alpha 1$ (VI) at day 7 and day 14, and less DCN at days 7 and 28.

To ensure viral treatment did not affect viability during the experiment, live-dead analysis was performed at 7 , 14 and $28 \mathrm{~d}$ after hMSCs were embedded in alginate. Cells in all experimental groups maintained greater than $50 \%$ viability through the $28 \mathrm{~d}$. There was no significant difference between non-infected and infected cells; therefore, the viral transduction did not affect cell viability (data not shown).

To determine whether viral transduction or knockdown of colbal and dcn might disrupt chondrogenesis, we analysed acan and sox 9 expression in non- infected and GFP-, shColVI- and shDcn- transduced cells. Previous work has shown that hMSCs cultured in alginate hydrogels in GM exhibit some features of chondrogenesis, with pericellular deposition of type VI collagen during the first $7 \mathrm{~d}$ of culture, but only with TGF- $\beta 3$ supplementation 


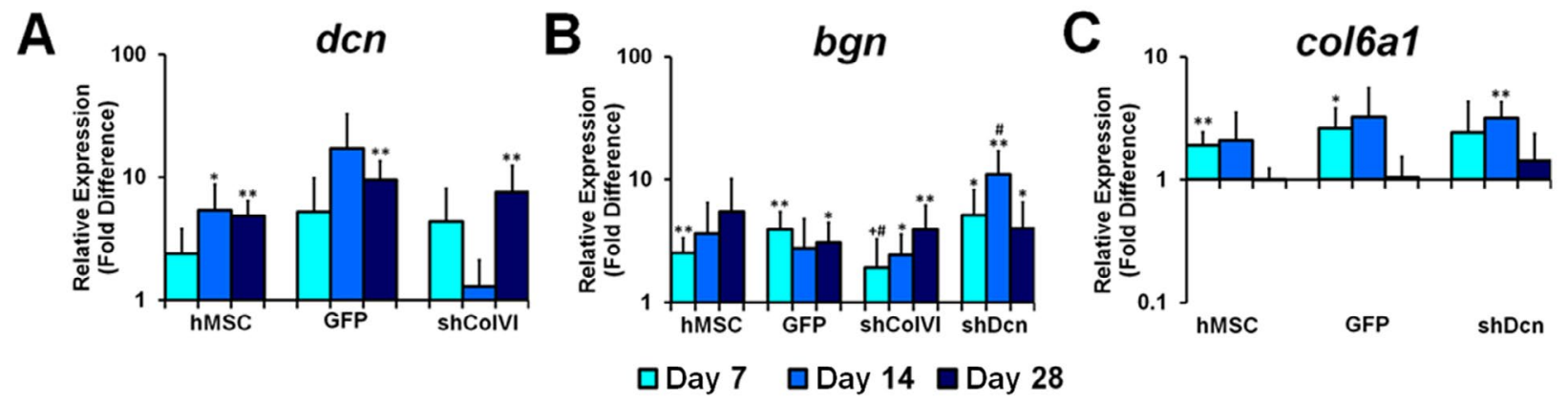

Fig. 5. Pericellular gene expression in control and knockdown cells. (A) Gene expression for $d c h$, (B) bgn and (C) col6al in non-infected, and GFP-, shColVI- and shDen- transduced hMSCs in alginate bead culture $(n=3)$ in CM relative to the same infection condition cultured in $\mathrm{GM}$ at 7,14 and $28 \mathrm{~d}\left({ }^{*} p<0.05, * * p<0.01\right.$, CM culture relative to same condition same time point in $\mathrm{GM} ;+p<0.05$, relative to CM-hMSCs at same time point; $\# p<0.05$, relative to CM-GFP hMSCs at same time point; $n \geq 3$ ). Data are shown as average values of the range of calculated fold differences $\left(2^{-\Delta \Delta \mathrm{Ct}+\mathrm{SD}}\right.$ and $\left.2^{-\Delta \Delta \mathrm{Ct}-\mathrm{SD}}\right) \pm$ range.

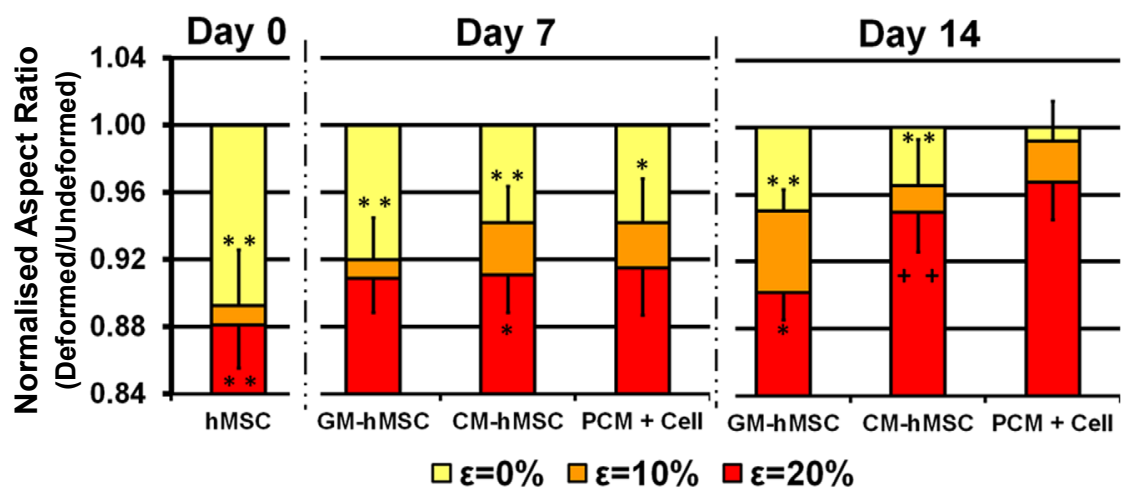

Fig. 6. Normalised aspect ratios (NAR) of cells cultured in GM or CM, and the PCM+Cell (CM-hMSCs). Each condition was subjected to 0,10 or $20 \%$ applied strain at 0,7 and $14 \mathrm{~d}$ of culture in alginate beads. Graphs show normalised aspect ratios that are overlaid with progressively increasing strain increments (values are not cumulative). ( $p<0.05$ and $p<0.01$ with respect to increasing symbols. *: from previous applied strain; +: from GM-hMSCs of same strain, same day; $n>30$ ). Data are shown as NAR \pm SEM.

did chondrogenesis progress fully (Vigfusdottir et al., 2010). Higher transcript levels were measured for both chondrogenic markers across all groups when cultured in $\mathrm{CM}$ at all time points relative to corresponding transduced cells cultured in GM (Fig. 3A and 3B). Differences were statistically significant only at particular time points, likely due to fluctuating levels of gene expression during differentiation. There was no significant difference between non-infected controls and knockdown samples at any time point for sox 9 expression. These data indicate that transduction with the silencing cassette did not affect the ability of hMSCs to undergo chondrogenic differentiation.

\section{Knockdown of col6al and $d c n$ alter PCM structure and gene regulation}

The organisation of the pericellular matrix is temporally dependent on its composition. DCN is a primary modulator of ColVI network assembly as well as a connector to type II collagen and aggrecan (Bidanset et al., 1992; Nareyeck et al., 2004; Wiberg et al., 2003). Targeted subtraction of ColVI and DCN were each expected to have direct impact on expression of pericellular proteins as well as PCM organisation during chondrogenesis.
ColVI and DCN deposition during chondrogenesis fully enveloped the non-infected and GFP-transduced cells (Fig. 4A and 4B). In non- and GFP-infected cells, DCN was visualised in concentrated pockets directly surrounding the cell membrane, with diffuse staining extending away from the cell mainly at day 7 and becoming more tightly gathered by day 28 (Fig. 4B as denoted by yellow arrows). This is consistent with the notion that DCN binds proteins within the PCM to form a fully developed network, controlling protein spacing with its attached GAG chain.

Knocking down ColVI resulted in lower acan, bgn, and $d c n$ levels during the first $14 \mathrm{~d}$ of chondrogenesis relative to non-infected and GFP-transduced cells (Fig. 3A, 5A and 5B). This difference was diminished by $28 \mathrm{~d}$. Visualisation of DCN surrounding shColVI cells did not reflect the decreased transcription, showing similar fluorescence to control cells. Although ColVI knockdown affected dcn expression, DCN knockdown did not affect col6al levels (Fig. 5C) but did alter its assembly. ColVI staining appeared to be membrane-bound and punctate, suggesting that DCN deficiency prevents ColVI from forming a continuous microfibrillar layer surrounding the cell (Fig. 4A as denoted by red arrows). This punctate staining was 
maintained over the course of the $28 \mathrm{~d}$ experiment. DCN knockdown also affected the expression of other PCM proteins, leading to increased levels of acan, bgn, and $\operatorname{sox} 9$ during the first two weeks. These results indicate that PCM gene expression is cross-regulated, with the greatest effects being in the first two weeks of chondrogenesis.

\section{Cellular and PCM stiffnesses evolve during chondrogenic differentiation}

In an effort to understand the mechanical role of the developing PCM during chondrogenesis, we employed an approach to quantify both the deformation of the cell itself (CM-hMSC) and the aggregate deformation of the cell with its PCM (PCM+Cell) over time in chondrogenic media (Fig. 6). Day 0 baseline controls represent hMSCs that had been embedded in alginate constructs directly from monolayer culture, and consequently had no exposure to chondrogenic media. At this time point, significant changes in normalised aspect ratios (NARs) were observed with applied strain. Note the nonlinear cell stiffness at day 0 ; cells deform greater from 0 to $10 \%$ applied strain than from 10 to $20 \%$.

At Day 7, GM cells (GM-hMSC) deformed less than Day 0 hMSCs, likely due to matrix production and assembly stimulated by 3D culture (Vigfusdottir et al., 2010) which is relatively stable between day 7 and day 14. Because there was no discernible PCM around GM cells, deformations were measured for cells only. For the $\mathrm{CM}$ group, incomplete elaboration of the PCM resulted in similar deformations between the cell (CM-hMSC) and the $\mathrm{PCM}+$ Cell.

At Day 14, GM cells exhibited significantly less deformation than at Day 7, but only at $10 \%$ applied strain. For CM cells, the aggregate deformation of $\mathrm{PCM}+\mathrm{Cell}$ was also correspondingly lower than at Day 7 . The most striking change was in the PCM+Cell deformation, which was significantly lower at both 10 and $20 \%$ applied strain. Interestingly, under $10 \%$ strain, the change in NAR for $\mathrm{CM}$ cells was higher than that for the $\mathrm{PCM}+\mathrm{Cell}$, but these trends are reversed at $20 \%$ applied strain (Fig.1B). This suggests that the PCM is stiffer than the cell at $10 \%$ applied strain, but that the cell becomes relatively stiffer than the PCM at $20 \%$.

ColVI and DCN are essential for resisting cellular deformation during compression

Our results above are consistent with previous research findings that during chondrogenesis hMSCs develop a mechanically functional PCM (Knight et al., 1998; Vigfusdottir et al., 2010), and that changes in its stiffness affects cell deformation (Lee et al., 2010). As a step toward elucidating the functional roles of specific PCM proteins, we examined cell deformations in genetically engineered CM-hMSCs. Again, for baseline measurements (Day 0), we seeded transduced and control cells into alginate discs, and immediately subjected them to compressive loading. Because of the universal lack of PCM, cells exhibited significant changes in normalised aspect ratios with each applied strain, with no significant differences between groups (Fig. 7A). Similar nonlinearity in cell stiffness as
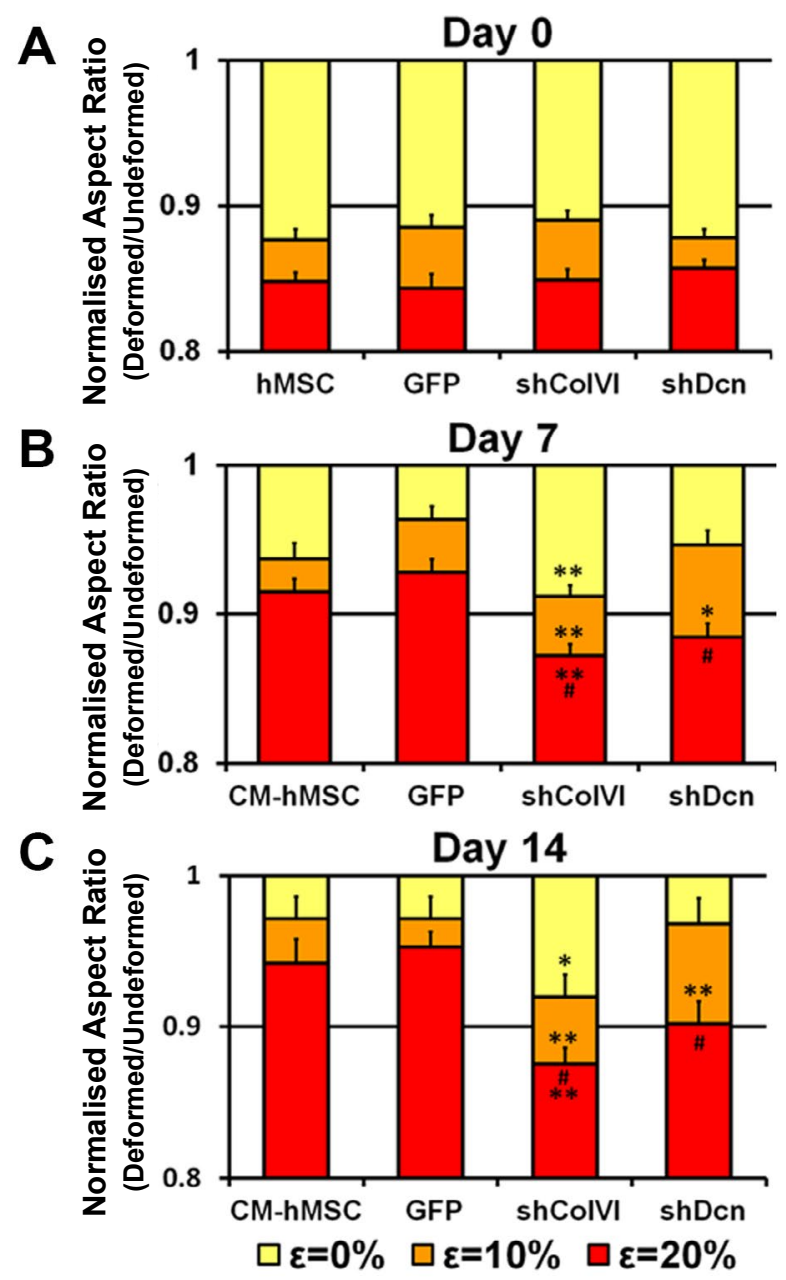

Fig. 7. Normalised aspect ratios of non-infected and GFP-, shColVI-, and shDcn-transduced hMSCs. Each condition was subjected to 0,10 and $20 \%$ applied strain at (A) day $0,(\mathbf{B})$ day 7 , and (C) day 14. Graphs show normalised aspect ratios that are overlaid with progressively increasing strain increments (values are not cumulative) $(* p<0.05, * * p<0.01$ significant difference from GFP-transduced cells at same nominal strain; $\# p<0.05$ significant deformation between 10 and $20 \%$ applied nominal strain; $n>30$ ). Data are shown as $\mathrm{NAR} \pm \mathrm{SEM}$.

observed in the previous experiment (Fig. 6) was found across all groups.

With culture, all conditions showed a greater resistance to the applied compression. However, the extent to which cell deformations decreased was dependent on treatment. Both non-infected CM-hMSCs and GFP controls exhibited the greatest decreases in cell deformation at both Day 7 and Day 14. This was expected since the PCM was allowed to develop normally in both groups. In addition, their similarity to each other confirms that the lentiviral infection of hMSCs does not affect cell stiffness.

ColVI deficient cells showed the greatest deformations across all groups at both Day 7 and Day 14, and for both magnitudes of applied strain (Fig. 7B and 7C). Deformation was similar between Days 7 and 14, indicating that the 
Table 3: Normalised AR values of each deformation and condition. ( $p<0.05$ and $p<0.01$ with respect to increasing symbols. \#: from Day 7 same strain, + : from D0 same strain. $n>30$ ).

\begin{tabular}{|l|l|l|l|l|}
\hline & \multicolumn{2}{|l|}{$\boldsymbol{\varepsilon}=\mathbf{1 0} \%$} & $\boldsymbol{\varepsilon}=\mathbf{2 0} \%$ \\
\hline Day 0 & $0.893 \pm 0.032$ & & $0.881 \pm 0.026$ & \\
\hline Day 7 GM & $0.920 \pm 0.025$ & $(\dagger)$ & $0.909 \pm 0.021$ & $(\dagger+)$ \\
\hline Day 14 GM & $0.950 \pm 0.013$ & $(\dagger+\#)$ & $0.902 \pm 0.017$ & $(\dagger)$ \\
\hline Day 7 CM & $0.942 \pm 0.022$ & $(\dagger)$ & $0.911 \pm 0.022$ & $(\dagger+$ \\
\hline Day 14 CM & $0.965 \pm 0.028$ & $(\dagger)$ & $0.949 \pm 0.024$ & $(\dagger+\# \#)$ \\
\hline Day 7 PCM+Cell & $0.942 \pm 0.026$ & & $0.915 \pm 0.028$ & \\
\hline Day 14 PCM+Cell & $0.992 \pm 0.023$ & $(\# \#)$ & $0.968 \pm 0.023$ & $(\#)$ \\
\hline
\end{tabular}

cell's inability to put in place a mechanical barrier does not change with time. Although direct comparisons cannot be made, we measured greater shColVI cell deformations than GM-hMSCs in previous experiments (Fig. 6).

Knocking down DCN resulted in interesting straindependent trends in cell deformation that were similar between Days 7 and 14 (Fig. 7B and 7C). At low levels of applied strain, cells deformed comparably with noninfected CM-hMSC and GFP controls, suggesting that the PCM was mechanically robust enough to sustain similar loads. However, once the applied strain reached $20 \%$, cellular resistance to deformation collapsed. We attribute this behaviour to the functional significance of decorin in mediating collagen-collagen interactions.

Overall, these results demonstrate that both ColVI and DCN have critical biophysical roles in regulating cell shape. In particular, ColVI is required for resisting even low magnitude strains, while DCN is important in maintaining structural integrity at higher strains.

\section{Discussion}

Stem cell therapies are currently being explored for their potential in the regeneration of load-bearing tissues, such as cartilage, due to both their pluripotency and their ability to maintain a basal phenotype during expansion in vitro. The PCM developed during chondrogenesis has been considered vital to the regulation of mechanotransduction events in differentiating hMSCs (Alexopoulos et al., 2003; Guilak et al., 2006; Villanueva et al., 2009; Vincent, 2013). Reconstruction of the PCM in isolated mature chondrocytes has been shown to be complex, temporally specific in composition, and highly dependent on proteinprotein interactions within the PCM (Knight et al., 1998; Quinn et al., 2002; DiMicco et al., 2007). Because chondrocyte function is closely tied to interactions with ECM proteins (Lee et al., 2000), altering the constituents of the PCM would likely induce cascading effects on cellcell and cell-matrix interactions. PCM development in chondrogenic hMSCs is expected to be even more complex, due to the additional changes in gene regulation associated with differentiation. To elucidate the functional roles of individual components in the PCM, we investigated the consequences of knocking down col6al and dcn mRNA expression using shRNA lentiviral vectors.

Type VI collagen is a $125 \mathrm{~nm}$ long heterotrimer that consists of three different $\alpha$-chains ( $\alpha 1(\mathrm{VI}), \alpha 2(\mathrm{VI})$ and $\alpha 3(\mathrm{VI}))$ and self-assembles into beaded filaments and larger fibrillar structures (Bruns et al., 1986). ColVI trimer formation occurs intracellularly before being secreted into the PCM for microfibrillar networking via $\mathrm{BGN}$ or DCN (Bruns et al., 1986; Engvall et al., 1986; Wiberg et al., 2003; DiMicco et al., 2007) and is maintained at low levels of transcription (DiMicco et al., 2007; Vigfusdottir et al., 2010). DCN is a ubiquitous proteoglycan, consisting of a core protein with a single chondroitin sulphate (Li et al., 2010) or dermatan sulphate (Knudson et al., 1996) side chain. It interacts with multiple collagens to create functional bridges between the PCM and surrounding ECM, and is involved in controlling fibrillogenesis and growth factor bioavailability (Chen et al., 2009; Lewis et al., 2010; Zhang et al., 2011). This study shows that these two specific proteins, ColVI and DCN, have profound influence over PCM composition and biomechanical behaviour surrounding hMSCs undergoing chondrogenesis.

One of our major findings was the significant contributions of ColVI and DCN in cellular resistance to deformation. A developing PCM has been implicated in acting as both a biomechanical buffer (Knight et al., 1998; Vigfusdottir et al., 2010; Appelman et al., 2011) as well as a biochemical bridge (Guilak et al., 2006; Villanueva et al., 2009; Vincent, 2013). Acting as a biomechanical buffer, the PCM shields the cell from deformation which could be potentially detrimental while also enhancing small tissue strains for stimulation (Alexopoulos et al., 2005; Choi et al., 2007; Vigfusdottir et al., 2010). Previous studies analysing deformation properties of chondrocytes in alginate hydrogels have shown that cells under compression form oblate spheroids with a decrease in cell diameter along the axis of loading and an increase in transverse cell diameters perpendicular to that axis (Knight et al., 2002). During initial matrix deposition, we found that the matrix and cell share similar deformations, due to the lack of full matrix envelopment. As the matrix stiffens to approach and then surpass cellular stiffness, the mechanical properties are contributed to more equally by both components (Lee et al., 2010). With a fully developed matrix, at higher strains the $\mathrm{PCM}+\mathrm{Cell}$ would reorganise due to hydration changes and spatial consolidation of collagen. Studies examining the PCM surrounding mechanically isolated chondrons are consistent with an initial elastic response followed by a transiently changing flow-dependent creep response under applied stress (Alexopoulos et al., 2005; Alexopoulos et al., 2009). The mechanical behaviour is dependent on PCM structure, with the elastic response governed by matrix 
deposition (Lee et al., 2010) that is highly associated with sGAG and collagen accumulation (Knight et al., 1998), and the viscous response governed by the osmotic and porous properties of the PCM network (McLane et al., 2013).

By altering the presence of ColVI and DCN, we sought to determine their involvement in this composition dependent mechanical response. In mature articular chondrocytes, the PCM has a higher modulus than that of the cell (Guilak et al., 1999). Previous studies in ColVInull mice demonstrate that a PCM can be formed, but that ColVI-null chondrons have significantly reduced stiffness compared to wild-type chondrons (Alexopoulos et al., 2009). The lowered resistance to deformation we observed in ColVI knockdown cells during chondrogenic PCM development is consistent with this reduced stiffness. ColVI can therefore be considered the primary protein involved in buffering loads to differentiating hMSCs.

During PCM development, it has been seen that sGAGs are important in the initial stages of assembly, directly impacting cellular mechanical responses to load (Knight et al., 1998). PGs mediate the PCM to ECM interface through interactions with types II and VI collagen (Bidanset et al., 1992; Wiberg et al., 2003; Connelly et al., 2008; Chen et al., 2009). Cells compensated for $d c n$ knockdown by upregulating $b g n$ expression, demonstrating a regulatory effect by DCN on other PCM small leucine rich PGs (sLRPs) (Ferdous et al., 2007). BGN core protein has significant sequence homology with that of DCN, but is structurally distinct and possesses two (instead of one) CS/DS chains (Fisher et al., 1989; Neame et al., 1989; Krishnan et al., 1999). Similar compensatory effects have also been seen in DCN knockout models (Chen et al., 2009; Lewis et al., 2010). BGN shares binding sites on collagen proteins with DCN. However, its lower affinity for these binding sites results in less involvement in PCM and ECM organisation (Chen et al., 2009), and its extra CS/DS- side chain results in a looser collagen network (Bidanset et al., 1992; Wiberg et al., 2001; Nareyeck et al., 2004; Ferdous et al., 2007; Lewis et al., 2010). The looser collagen spacing possibly contributed to the significant increases in shDen cellular deformation at higher strains. This network is bound to the cell membrane through HA core proteinCD44 interactions (Knudson et al., 1996), NG2 receptors (membrane bound chondroitin sulphate PGs) or through direct binding to the $\alpha 1 \beta 1$ integrin (Loeser et al., 2000). From our confocal images, the altered ColVI assembly in shDcn knockdown cells appeared as punctate localisations completely surrounding the cells at these binding sites.

In addition to acting as a mechanical stabiliser, the PCM also aids maintenance of chondrocyte phenotype biochemically. Our data are in agreement with chondrogenic stage specific gene expression ( $\mathrm{Xu}$ et al., 2008), with col6al being significantly greater in the first seven days of chondrogenesis in non-infected and GFP-transduced cells, which decreases in expression by $28 \mathrm{~d}$ of culture. $\mathrm{DCN}$ is involved in the sequestration of TGF- $\beta 3$, FGF-2, TNF- $\alpha$, PDGF, and IGF-1 (Ferdous et al., 2007; Chen et al., 2009; Zhang et al., 2011) and has been associated with cell proliferation signalling (Chen et al., 2009), causing the cell to cease proliferation and enhance matrix synthesis.
These growth factors cause PCM accumulation during chondrogenesis in a concentration dependent manner (Mehlhorn et al., 2006; Lee et al., 2010; Li et al., 2010). Sequestration of growth factors immediately surrounding the cell allows for specifically stimulated responses, being released mechanically or enzymatically from their associated PG for presentation to the cell membrane. Sox9, comp and HAPLN1 expression levels have shown to reach a peak by $12 \mathrm{~d}$ of culture associated with high levels of matrix assembly and metabolism (Xu et al., 2008). The transient overexpression of $\operatorname{sox} 9$ in $\operatorname{shDcn}$ cells at day 14 could be due to the lack of regulation and continuous bioavailability of TGF- $\beta 3$ to the cell.

Full PCM formation occurs within the first two weeks of TGF- $\beta 3$ stimulation in vitro, which parallels the upregulation of acan and bgn expression during the first $14 \mathrm{~d}$, and then steadily matures as acan returns to control levels. PCM retention has been shown to be mediated through interactions between CD44 and hyaluronic acid, which lay a foundation for aggrecan accumulation and affects PCM volume and shape (Alexopoulos et al., 2005; Knudson et al., 1996). Hyaluronan is critical for maintenance of the pericellular environment surrounding chondrocytes (Lee et al., 2000), interacting with type II collagen, aggrecan and link protein. The observed higher acan upregulation in shDen cells could aid in matrix protein retention and reorganisation, causing large changes in the mechanical composition of the PCM, affecting cellular metabolism. shColVI cells showed an opposite trend of acan and bgn expression, with lower expression levels during the first $14 \mathrm{~d}$ of culture than all other samples.

\section{Conclusion}

To our knowledge, this study is the first to elucidate the functional significance of DCN in the PCM. Furthermore, we extend our previous work (Vigfusdottir et al., 2010) by demonstrating that ColVI is crucial for the biomechanical integrity of the PCM in chondrogenic hMSCs at all stages of differentiation. The use of shRNA expressing lentiviral vectors allows stable production of siRNAs by the cell (Dykxhoorn et al., 2003). This approach has enabled us to characterise how specific PCM proteins govern the micromechanical environment of differentiating hMSCs over time. Although further studies are required to elucidate the downstream effects on mechanotransduction events, signalling cascades, and load-induced behaviours, our results provide some immediate insight into strategies that can be used to engineer specific microenvironments for eliciting desired cellular responses.

\section{Acknowledgements}

This work was supported funded by support by the National Science Foundation (CBET-0845754; AHH), the University of Maryland, College Park (JDT), and the Howard Hughes Medical Institute (PIT). 


\section{References}

Alexopoulos LG, Haider MA, Vail TP, Guilak F (2003) Alterations in the mechanical properties of the human chondrocyte pericellular matrix with osteoarthritis. J Biomech Eng 125: 323-333.

Alexopoulos LG, Williams GM, Upton ML, Setton LA, Guilak F (2005) Osteoarthritic changes in the biphasic mechanical properties of the chondrocyte pericellular matrix in articular cartilage. J Biomech 38: 509-517.

Alexopoulos LG, Youn I, Bonaldo P, Guilak F (2009) Developmental and osteoarthritic changes in Col6a1knockout mice biomechanics of type VI collagen in the cartilage pericellular matrix. Arthritis Rheum 60: 771-779.

Appelman TP, Mizrahi J, Seliktar D (2011) A finite element model of cell-matrix interactions to study the differential effect of scaffold composition on chondrogenic response to mechanical stimulation. J Biomech Eng 133: 041010.

Bidanset DJ, Guidry C, Rosenberg LC, Choi HU, Timpl R, Hook M (1992) Binding of the proteoglycan decorin to collagen type-VI. J Biol Chem 267: 5250-5256.

Bruns RR, Press W, Engvall E, Timpl R, Gross J (1986) Type-VI collagen in extracellular, $100 \mathrm{nM}$ periodic filaments and fibrils- identification by immunoelectron microscopy. J Cell Biol 103: 393-404.

Chen WH, Lai MT, Wu ATH, Wu CC, Gelovani JG, Lin CT, Hung SC, Chiu WT, Deng WP (2009) In vitro stage-specific chondrogenesis of mesenchymal stem cells committed to chondrocytes. Arthritis Rheum 60: 450-459.

Choi JB, Youn I, Cao L, Leddy HA, Gilchrist CL, Setton LA, Guilak F (2007) Zonal changes in the three-dimensional morphology of the chondron under compression: The relationship among cellular, pericellular, and extracellular deformation in articular cartilage. J Biomech 40: 2596-2603.

Connelly JT, Wilson CG, Levenston ME (2008) Characterization of proteoglycan production and processing by chondrocytes and BMSCs in tissue engineered constructs. Osteoarthritis Cartilage 16: 1092-1100.

DiMicco MA, Kisiday JD, Gong H, Grodzinsky AJ (2007) Structure of pericellular matrix around agaroseembedded chondrocytes. Osteoarthritis Cartilage 15: 1207-1216.

Doane KJ, Howell SJ, Birk DE (1998) Identification and functional characterization of two type VI collagen receptors, alpha(3)beta(1) integrin and NG2, during avian corneal stromal development. Invest Ophthalmol Vis Sci 39: 263-275.

Dykxhoorn DM, Novina CD, Sharp PA (2003) Killing the messenger: Short RNAs that silence gene expression. Nat Rev Mol Cell Biol 4: 457-467.

Engvall E, Hessle H, Klier G (1986) Molecular assembly, secretion, and matrix deposition of type-VI collagen. J Cell Biol 102: 703-710.

Ferdous Z, Wei VM, Iozzo R, Hook M, Grande-Allen KJ (2007) Decorin-transforming growth factor-beta interaction regulates matrix organization and mechanical characteristics of three-dimensional collagen matrices. J Biol Chem 282: 35887-35898.
Fisher LW, Termine JD, Young MF (1989) Deduced protein sequence of bone small proteoglycan I (biglycan) shows homology with proteoglycan II (decorin) and several nonconnective tissue proteins in a variety of species. J Biol Chem 264: 4571-4576.

Guilak F, Jones WR, Ting-Beall HP, Lee GM (1999) The deformation behavior and mechanical properties of chondrocytes in articular cartilage. Osteoarthritis Cartilage 7: 59-70.

Guilak F, Alexopoulos LG, Upton ML, Youn I, Choi JB, Cao L, Setton LA, Haider MA (2006) The pericellular matrix as a transducer of biomechanical and biochemical signals in articular cartilage. Ann N Y Acad Sci 1068: 498-512.

Keene DR, Engvall E, Glanville RW (1988) Ultrastructure of type-VI collagen in human-skin and cartilage suggests an anchoring function for this filamentous network. J Cell Biol 107: 1995-2006.

Knight MM, Lee DA, Bader DL (1998) The influence of elaborated pericellular matrix on the deformation of isolated articular chondrocytes cultured in agarose. Biochim Biophys Acta 1405: 67-77.

Knight MM, Ross JM, Sherwin AF, Lee DA, Bader DL, Poole CA (2001) Chondrocyte deformation within mechanically and enzymatically extracted chondrons compressed in agarose. Biochim Biophys Acta 1526: 141146.

Knight MM, Bravenboer J, Lee DA, van Osch G, Weinans H, Bader DL (2002) Cell and nucleus deformation in compressed chondrocyte-alginate constructs: temporal changes and calculation of cell modulus. Biochim Biophys Acta 1570: 1-8.

Knudson W, Aguiar DJ, Hua Q, Knudson CB (1996) CD44-anchored hyaluronan-rich pericellular matrices: An ultrastructural and biochemical analysis. Exp Cell Res 228: 216-228.

Krishnan P, Hocking AM, Scholtz JM, Pace CN, Holik KK, McQuillan DJ (1999) Distinct secondary structures of the leucine-rich repeat proteoglycans decorin and biglycan. Glycosylation-dependent conformational stability. J Biol Chem 274: 10945-10950.

Lee B, Han L, Frank EH, Chubinskaya S, Ortiz C, Grodzinsky AJ (2010) Dynamic mechanical properties of the tissue-engineered matrix associated with individual chondrocytes. J Biomech 43: 469-476.

Lee V, Cao L, Zhang Y, Kiani C, Adams ME, Yang BB (2000) The roles of matrix molecules in mediating Chondrocyte aggregation, attachment, and spreading. J Cell Biochem 79: 322-333.

Lewis JL, Krawczak DA, Oegema TR, Westendorf JJ (2010) Effect of decorin and dermatan sulfate on the mechanical properties of a neocartilage. Connect Tissue Res 51: 159-170.

Li Z, Kupcsik L, Yao SJ, Alini M, Stoddart MJ (2010) Mechanical load modulates chondrogenesis of human mesenchymal stem cells through the TGF-beta pathway. J Cell Mol Med 14: 1338-1346.

Livak KJ, Schmittgen TD (2001) Analysis of relative gene expression data using real-time quantitative PCR and the 2(T)(-Delta Delta C) method. Methods 25: 402-408. 
Loeser RF, Sadiev S, Tan L, Goldring MB (2000) Integrin expression by primary and immortalized human chondrocytes: evidence of a differential role for alpha 1 beta 1 and alpha 2 beta 1 integrins in mediating chondrocyte adhesion to types II and VI collagen. Osteoarthritis Cartilage 8: 96-105.

McLane LT, Chang P, Granqvist A, Boehm H, Kramer A, Scrimgeour J, Curtis JE (2013) Spatial organization and mechanical properties of the pericellular matrix on chondrocytes. Biophys J 104: 986-996.

Mehlhorn AT, Schmal H, Kaiser S, Lepski G, Finkenzeller G, Stark GB, Suekamp NP (2006) Mesenchymal stem cells maintain TGF-beta-mediated chondrogenic phenotype in alginate bead culture. Tissue Eng 12: 1393-1403.

Nareyeck G, Seidler DG, Troyer D, Rauterberg J, Kresse H, Schonherr E (2004) Differential interactions of decorin and decorin mutants with type I and type VI collagens. Eur J Biochem 271: 3389-3398.

Neame PJ, Choi HU, Rosenberg LC (1989) The primary structure of the core protein of the small, leucine-rich proteoglycan (PG I) from bovine articular cartilage. J Biol Chem 264: 8653-8661.

Poole CA, Ayad S, Gilbert RT (1992) Chondrons from articular-cartilage: Immunohistochemical evaluation of type-VI collagen organization isolated chondrons by light, confocal and electron-microscopy. J Cell Sci 103: 11011110 .

Quinn TM, Schmid P, Hunziker EB, Grodzinsky AJ (2002) Proteoglycan deposition around chondrocytes in agarose culture: construction of a physical and biological interface for mechanotransduction in cartilage. Biorheology 39: 27-37.

Rastogi A, Thakore P, Leung A, Benavides M, Machado M, Morschauser MA, Hsieh AH (2009) Environmental regulation of notochordal gene expression in nucleus pulposus cells. J Cell Physiol 220: 698-705.

Rutledge RG, Cote C (2003) Mathematics of quantitative kinetic PCR and the application of standard curves. Nucl Acids Res 31: e93.

Stewart SA, Dykxhoorn DM, Palliser D, Mizuno H, Yu EY, An DS, Sabatini DM, Chen ISY, Hahn WC, Sharp PA, Weinberg RA, Novina CD (2003) Lentivirus-delivered stable gene silencing by RNAi in primary cells. RNA-Publ. RNA Soc 9: 493-501.

Vigfusdottir AT, Pasrija C, Thakore PI, Schmidt RB, Hsieh AH (2010) Role of pericellular matrix in mesenchymal stem cell deformation during chondrogenic differentiation. Cell Mol Bioeng 3: 387-397.

Villanueva I, Weigel CA, Bryant SJ (2009) Cell-matrix interactions and dynamic mechanical loading influence chondrocyte gene expression and bioactivity in PEG-RGD hydrogels. Acta Biomater 5: 2832-2846.

Vincent TL (2013) Targeting mechanotransduction pathways in osteoarthritis: a focus on the pericellular matrix. Curr Opin Pharmacol 13: 449-454.

Vonk LA, Doulabi BZ, Huang CL, Helder MN, Everts V, Bank RA (2010) Preservation of the chondrocyte's pericellular matrix improves cell-induced cartilage formation. J Cell Biochem 110: 260-271.
Wang P, Yang L, Hsieh AH (2011) Nucleus pulposus cell response to confined and unconfined compression implicates mechanoregulation by fluid shear stress. Ann Biomed Eng 39: 1101-1111.

Wiberg C, Hedbom E, Khairullina A, Lamande SR, Oldberg A, Timpl R, Morgelin M, Heinegard D (2001) Biglycan and decorin bind close to the N-terminal region of the collagen VI triple helix. J Biol Chem 276: $18947-$ 18952.

Wiberg C, Klatt AR, Wagener R, Paulsson M, Bateman JF, Heinegard D, Morgelin M (2003) Complexes of matrilin-1 and biglycan or decorin connect collagen VI microfibrils to both collagen II and aggrecan. J Biol Chem 278: 37698-37704.

Xu JP, Wang W, Ludeman M, Cheng KV, Hayami T, Lotz JC, Kapila S (2008) Chondrogenic differentiation of human mesenchymal stem cells in three-dimensional alginate gels. Tissue Eng Part A 14: 667-680.

Zhang ZJ, Jin W, Beckett J, Otto T, Moed B (2011) A proteomic approach for identification and localization of the pericellular components of chondrocytes. Histochem Cell Biol 136: 153-162.

\section{Web References}

Web Ref. $1<$ http://cancan.cshl.edu/cgi-bin/Codex/Codex. cgi> [10-06-2009]

Web Ref. $2<$ http://frodo.wi.mit.edu $>$ [01-07-2009]

\section{Discussion with Reviewers}

P. Pravincumar: Could you propose specific suggestions on how we could control the stem cell (mechanobiology) differentiation based on the presence or not of the two studied pericellular matrix proteins?

Authors: This current study establishes the feasibility and effectiveness of using RNA interference as a technique to engineer the cellular biophysical microenvironment. Specifically, we demonstrate that ColVI and DCN knockdown can have a profound effect on how cells deform, during TGF $\beta$-induced chondrogenic differentiation. During dynamic loading, forces are transmitted from the PCM, across the cell membrane, along the cytoskeleton, to the nucleus, and ultimately elicit a biochemical response (Guilak, 1995, additional reference; Lee et al., 2000, text reference).

The ability to modulate the outside-in signalling cascade provides us with a means to control stem cell differentiation and mechanobiology, in a way that enhances the traditional respective approaches using growth factor stimulation and loading. This level of control can be harnessed to augment current strategies in regenerative medicine. For instance, we postulate that modulating the expression of ColVI and DCN can effectively influence the mechanobiological regulation of cellular differentiation in either naïve stem cells or lineage-specific progenitors. In naïve mesenchymal stem cells, it has been previously shown that biophysical stimuli, in the absence of biochemical differentiation factors, can mechanobiologically drive differentiation 
(Engler et al., 2006; Haudenschild et al., 2009, additional references). With ColVI knockdown, the inability for hMSCs to form a mechanically robust PCM results in greater degrees of distortional strain under compressive loading. We would expect these cells to exhibit impaired chondrogenic differentiation and enhanced fibrogenic/ osteogenic potential. Knocking down DCN would likely yield strain-dependent effects, since the ability for the PCM to attenuate cell deformation is lost at higher loads. Therefore, within a single construct, transduced and nontransduced cells would be able to respond differently, even while the construct is stimulated under uniform biochemical and biomechanical conditions.

For chondrogenic progenitors, changing the cell's resistance to loading will cause a shift in mechanotransduction events, possibly initiating a more catabolic response or a more osteoarthritic phenotype. In either case, the mechanobiology of engineered hMSC differentiation would have to be experimentally investigated, because of the interacting factors that mediate cell deformation, and the complex pathways that regulate differentiation. Current studies show that chemically altering the cytoskeleton can tilt the mechanoresponse of stem cells between chondrogenic versus hypertrophic differentiation (Guilak, 1995; Farquharson et al., 1999; Kumar and Lassar, 2009, additional references). Cytoskeletal alterations could also be indirectly achieved through ColVI and DCN knockdown, caused by changes in PCM-cell membrane interactions, though this also needs to be further explored. The composition of the PCM also has a bearing on the bioavailability of growth factors, which would further influence differentiation. DCN's ability to sequester TGF- $\beta$ within t)he PCM (Ferdous et al., 2007, text reference), for example, may control whether or not the stem cell's differentiation response is robust. Our study found that knocking down DCN resulted in a high upregulation of sox 9 at day 14 , which could indicate a temporal shift in terminal differentiation.

By targeted subtraction of ColVI and DCN, we can modify the cell's mechanobiological response either by controlling the ability of the cell to resist compression or by accelerating lineage- and stage-specific gene expression. Intrinsically regulating the cell's microenvironment allows the study of how these specific proteins are involved in short and long term mechanotransduction events that has yet to be achieved.

\section{Additional References}

Engler AJ, Sen S, Sweeney HL, Discher DE (2006) Matrix elasticity directs stem cell lineage specification. Cell 126: 677-689.

Farquharson C, Lester D, Seawright E, Jefferies D, Houston B (1999) Microtubules are potential regulators of growth-plate chondrocyte differentiation and hypertrophy. Bone 25: 405-412.

Guilak F (1995) Compression-induced changes in the shape and volume of the chondrocyte nucleus. J Biomech 28: $1529-1541$.

Haudenschild AK, Hsieh AH, Kapila S, Lotz JC (2009) Pressure and distortion regulate human mesenchymal stem cell gene expression. Ann Biomed Eng 37: 492-502.

Kumar D, Lassar AB (2009) The transcriptional activity of Sox 9 in chondrocytes is regulated by RhoA signaling and actin polymerization. Mol Cell Biol 29: 4262-4273. 